\title{
Phase clustering in complex networks of delay-coupled oscillators
}

\author{
Toni Pérez* \\ Physics Department, Lehigh University, Bethlehem, PA 18015. USA \\ Víctor M. Eguíluz \\ Instituto de Física Interdisciplinar y Sistemas Complejos IFISC (CSIC-UIB), E07122 Palma de Mallorca, Spain ${ }^{\dagger}$
}

\author{
Alex Arenas \\ Departament d'Enginyeria Informàtica i Matemàtiques, \\ Universitat Rovira $i$ Virgili, 43007 Tarragona, Spain
}

\begin{abstract}
We study the clusterization of phase oscillators coupled with delay in complex networks. For the case of difussive oscillators, we formulate the equations relating the topology of the network and the phases and frequencies of the oscillators (functional response). We solve them exactly in directed networks for the case of perfect synchronization. We also compare the reliability of the solution of the linear system for non-linear couplings. Taking advantage of the form of the solution, we propose a frequency adaptation rule to achieve perfect synchronization. We also propose a mean-field theory for uncorrelated random networks that proves to be pretty accurate to predict phase synchronization in real topologies, as for example the C.elegans or the Autonomous Systems connectivity.
\end{abstract}

Functional networks of complex systems are usually obtained monitoring the temporal activity of their components, and are often used to infer their unknown underlying connectivity. This process, of utmost importance, is analyzed here from a theoretical perspective. We take advantage of the mathematical simplicity of a diffusive system of coupled with delay oscillators, to analyze its temporal correlations and explore the relationship with the uderlying network topology. The study allows us to contribute to the understanding of the relation between topology and dynamics in complex networks, and provides useful connections between both, that can be used in the topological inference process using dynamical information.

\section{INTRODUCTION}

After Winfree's work [1], synchronization in populations of interacting elements has been a subject of intense research in physical, chemical, biological, and social systems [2-4]. Although Winfree's approach proved to be successful in describing the emergence of spontaneous synchronization in populations of oscillators, it was based on the premise that every oscillator feels the same mean-field. However, this implicit all-to-all connectivity between elements of a large population is difficult to imagine in real world. When the number of elements is large enough, this pattern is incompatible with physical constraints as for example minimization of energy (or costs), and in general with the rare observation of long

*Email: amp609@lehigh.edu

thttp://ifisc.uib-csic.es range interactions in systems formed by macroscopic elements. The particular local connectivity structure of the elements was missing (in fact, discarded) in these and subsequent approaches. Something similar occurs when considering the delays on their interactions.

There are plenty of studies of network synchrony in the absence of time delays [4]. However, interaction between elements usually involves the propagation through a communication channel, introducing delay. This delay is present in social and many biological systems [5], playing an essential role in neural networks [6]. The consideration of these delays is of utmost importance [7].

Recently, it has been analytically, and experimentally shown that zero time-lag synchronization is feasible over two distant (delayed) interacting oscillators when a third oscillator is placed in between of them $[8,9]$. A recent study sheds light along these lines by studying the synchronization of networks of chaotic units with timedelayed couplings using the formalism of the master stability function [10].

Our ansatz at this point is that the dynamics of a network of linear coupled oscillators with delays, should provide a simple toy model where to analyze relationships between the substrate of the interactions and the functional response of the system. There has been a big effort from the scientific community to infer the complex network of interactions between elements from the functional network [11-18]. We analyze the functional network resulting from the simplest dynamical system with delay presenting a synchronous dynamics, on a given topology. Given the simplicity of the model, we obtain the exact solution, develop a statistical mean-field theory approximation and find the relation between the degree distribution of the topological network and the associated functional network.

The paper is structured as follows, in section II we introduce the model and the general solution. In section 
III we explore the effect of the heterogeneity in the natural frequencies and the delays and we propose an adaptive frequency rule to obtain perfect synchronization. In section IV we compare both the lineal versus the nonlineal coupling. We also determine how the normalization of the coupling term changes the solution. In section $\mathrm{V}$ we develop a heterogeneous mean-field approximation and obtain a mean-field solution for directed uncorrelated networks. We illustrate this solution in two real networks like the neural network of the $C$. elegans and the Internet at the Autonomous System level. Finally, in section VI we present the main conclusions.

\section{OSCILLATORS' NETWORK MODEL}

We study a system of $N$ delay coupled oscillators which dynamics is described by:

$$
\dot{\phi}_{i}(t)=\omega_{i}+\sum_{j} a_{i j}\left(\phi_{j}\left(t-\tau_{i j}\right)-\phi_{i}(t)\right),
$$

where $\omega_{i}$ is the internal frequency of the $i$ th oscillator. The elements $a_{i j}$ of the adjacent matrix $\mathbf{A}$ define the network of interaction, that is, $a_{i j}=1$ if the oscillator $j$ influences $i$. We denoted the delay in the interaction by $\tau_{i j}$ indicating the time lag that oscillator $j$ needs to interact and affect the dynamics of oscillator $i$. It is worth noting that, if the phase differences remain small enough, Eq. (1) can be understood as the linearization of many nonlinear interaction models, including the Kuramoto model [19].

If the network of interactions presents a single component, Eq. (1) presents an unique phase locked solution of the form

$$
\phi_{i}(t)=\Omega t+\theta_{i}
$$

where $\Omega$ is the global locking frequency and $\theta_{i}$ the initial phase of oscillator $i$. Substituting in Eq. (1), we obtain a set of $N$ linear equations that can be written in matrix form as

$$
\boldsymbol{\omega}-\Omega(\mathbf{1}+\mathbf{T})=\mathbf{L} \boldsymbol{\theta}
$$

where $\boldsymbol{\omega}$ and $\boldsymbol{\theta}$ are the frequency vector and the phase vector with components $\omega_{i}$ and $\theta_{i}$ respectively. $\mathbf{L}$ is the Laplacian matrix defined as $L_{i j}=k_{i, \text { in }} \delta_{i j}-a_{i j}, k_{i \text {,in }}=$ $\sum_{j} a_{i j}$ is the in-degree of node $i, \delta_{i j}$ is the Kronecker delta, $\mathbf{1}$ is a vector of 1 's and $\mathbf{T}$ is a vector of components $T_{i}=\sum_{j} a_{i j} \tau_{i j}$ the total delay affecting each node.

In general, as the sum of the rows of the Laplacian matrix $\mathbf{L}$ is zero, there exist a left-eigenvector $\mathbf{c}=$ $\left(c_{1}, c_{2}, \ldots, c_{N}\right)$ with eigenvalue 0 , that is, $\mathbf{c L}=0$. This left-eigenvector $\mathbf{c}$ is unique as long as all the nodes of the network can be reached by at least one node [20]. Then, the locking frequency can be obtained by left-multiplying Eq. (3) by c

$$
\Omega=\frac{\langle\boldsymbol{\omega}\rangle}{1+\langle\mathbf{T}\rangle},
$$

where $\langle\mathbf{x}\rangle=\sum_{i} c_{i} x_{i}$ and $\mathbf{c}$ is normalized, $\sum_{i} c_{i}=1$. The phases are given by

$$
\frac{\omega_{i}-\langle\boldsymbol{\omega}\rangle+\left(\omega_{i}\langle\mathbf{T}\rangle-\langle\boldsymbol{\omega}\rangle T_{i}\right)}{1+\langle\mathbf{T}\rangle}=(\mathbf{L} \boldsymbol{\theta})_{i} .
$$

\section{UNDIRECTED NETWORKS: THE ROLE OF HETEROGENEITY}

Equations (4) and (5) allow us to investigate the relationship between the dispersion on distribution of delays, frequencies and phases. First, let's consider the solution of identical oscillators in undirected networks. In this case the oscillation frequency and the phases are given by

$$
\begin{gathered}
\Omega=\frac{\omega}{1+\langle k\rangle \tau}, \\
\frac{\omega \tau\left(\langle k\rangle-k_{i}\right)}{1+\langle k\rangle \tau}=(\mathbf{L} \boldsymbol{\theta})_{i} .
\end{gathered}
$$

When the frequencies or the delays are distributed, one can expect, due to the linear relationship found in Eq. (5), a distribution of the phases. Fig. 1 shows the distribution of individual phases $\theta_{i}$ for a network composed of $N=10^{3}$ nodes with a regular degree of $k_{i}=4$ when the frequencies or the delays are normally distributed. In Fig. 1(a) the natural frequencies are distributed following a Gaussian distribution with mean $\langle\boldsymbol{\omega}\rangle=1.0$ and standard deviation $\sigma_{\omega}=0.01$. In Fig. 1(b) the delays are Gaussian distributed with $\langle\boldsymbol{\tau}\rangle=0.1$ and $\sigma_{\tau}=0.01$. Using the same parameters, we observe that the distribution of delays has more impact in the dispersion of phases to achieve synchronization. Fig. 2 shows that the system exhibit a linear dependence in the standard deviation of the distribution of $\theta_{i}$ with respect to the standard deviation of the distribution of frequencies $\sigma\left(\omega_{i}\right)$ and delays $\sigma\left(\tau_{i}\right)$. The slope $A$ of the linear fitting in Fig. 2 provides the relationship between the dispersion of the phases and the mean degree. We found that the dispersion of the phases $\sigma\left(\theta_{i}\right)$ is related with the inverse of the mean degree $\langle k\rangle$ following $\sigma\left(\theta_{i}\right)=B /\langle k\rangle^{p}$ (see Fig. 3).

\section{A. Perfect Synchronization}

The formal solution obtained in Eq. (5) allows to explore the conditions in which the frequency, delays and topology can be combined to achieve perfect synchronization between each node. It is straightforward to obtain from Eq. 3 that the condition for fully clustered solution in the network $\left(\theta_{i}=\theta_{j}, \forall i, j\right)$ requires

$$
\frac{\omega_{i}}{\left(1+T_{i}\right)}=\Omega
$$

From Eq. (8) we see that in absence of delays $\left(T_{i}=0\right)$ all the elements must have the same natural frequency, and 

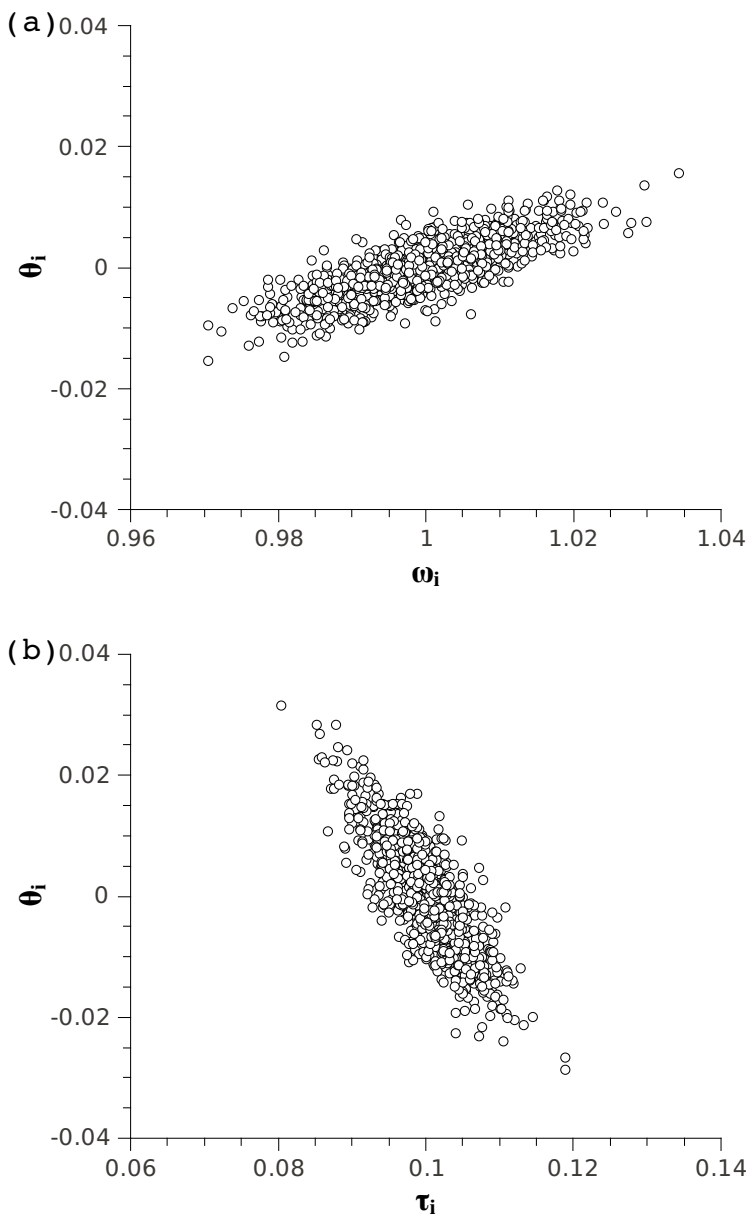

FIG. 1. Dispersion of the individual phases $\theta_{i}$ in a degree regular network versus (a) the natural frequency $\omega_{i}$ of the oscillators $\left(\langle\boldsymbol{\omega}\rangle=1.0, \sigma_{\omega}=0.01\right.$ and $\left.\tau_{i j}=0.1\right)$ and $(\mathrm{b})$ the delay $\tau_{i j}$ in their connections $\left(\langle\boldsymbol{\tau}\rangle=0.1, \sigma_{\tau}=0.01\right.$ and $\left.\omega_{i}=1.0\right)$.

reversely, if all nodes have the same natural frequency $\left(\omega_{i}=\omega\right)$, the total delay affecting each node must be the same. In the case of degree regular networks with identical elements and equal delays, i.e., $\omega_{i}=\omega$ and $\tau_{i}=\tau$, the frequency of the phase synchronized state is $\Omega=\frac{\omega}{1+k \tau}$. In general, it is always possible to choose the frequencies, the topology and the delays such that perfect coordinated activity is reached.

Note that once the dynamics starts, correlations arise between any two nodes in the network with different significance in their values ranging from 0 to 1 . The representation of the functional network we use will be always a weighted complete network, where the links weights represent the correlation between them. The functional network, that is, the network formed connecting those nodes displaying correlated activity, will be a fully connected network despite the sparse connectivity of the underlying interaction network.

Next, we explore the possibility to obtain perfect syn-
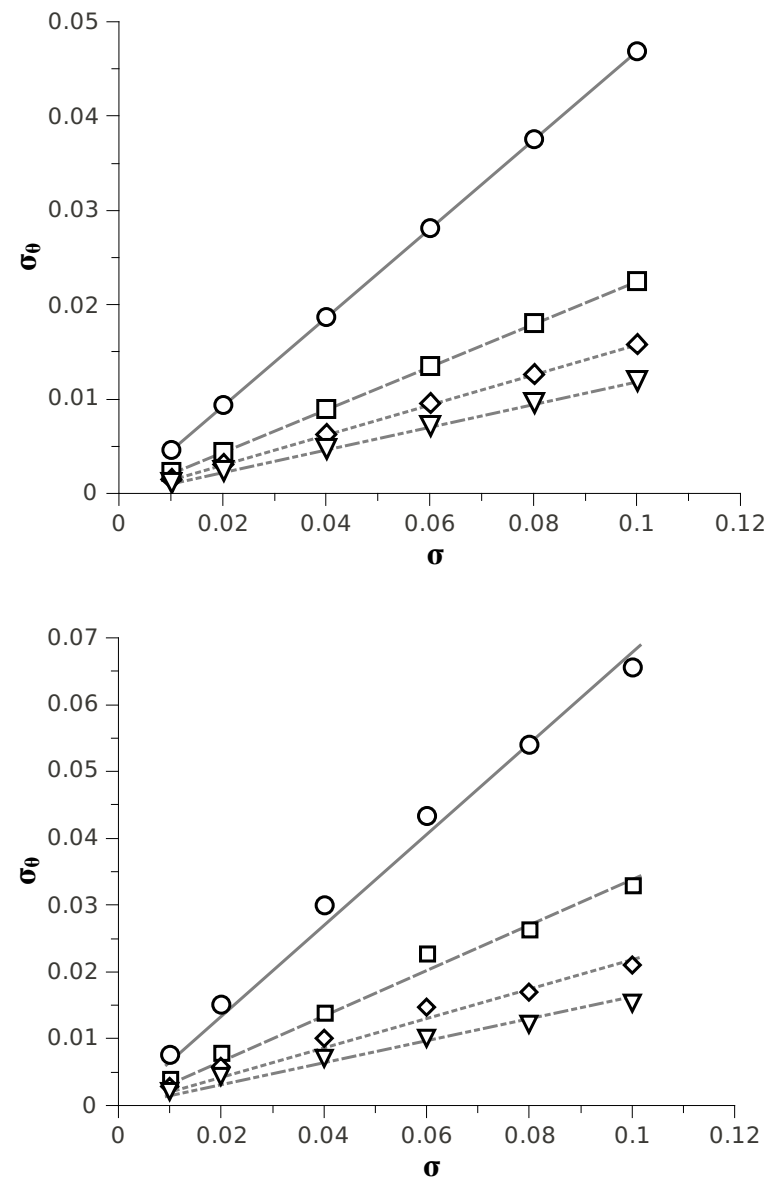

FIG. 2. Dependence of $\sigma\left(\theta_{i}\right)$ versus $\sigma\left(\omega_{i}\right)$ (upper panel) and $\sigma\left(\tau_{i}\right)$ (bottom panel) for different values of the mean degree $\langle k\rangle=4$ (circles), 6 (squares), 8 (diamonds) and 10 (triangles). The mean value of the distributions in each case is $\langle\boldsymbol{\omega}\rangle=1.0$ and $\langle\boldsymbol{\tau}\rangle=0.1$. Lines correspond to a lineal fitting $y=A x$.

chronization in an Erdös-Rényi (ER) network with $N=$ $10^{3}$ nodes and $\langle k\rangle=6$ by mean of a dynamical adjustment of the frequencies $\omega_{i}$. Initially, we start with a given distribution of frequencies for the nodes, then, we integrate Eq. (1) for a period of time after which the frequencies are readjusted proportionally to the difference between the phase of each node $\theta_{i}$ and the mean phase value $\langle\theta\rangle$ as follows: $\omega_{i}^{\prime}=\omega_{i}\left(1+\left(\langle\theta\rangle-\theta_{i}\right)\right)$. In the last iteration, as an alternative way to obtain perfect synchronization, we follow the recipe given in Eq. (8) and distribute the frequencies according to $\omega_{i}=\Omega\left(1+T_{i}\right)$ instead. In Fig. 4(a) we show the dispersion of the phases $\theta_{i}$ and the difference between the theoretical frequency $\omega_{t h}=\Omega\left(1+T_{i}\right)$ and the current frequency $\omega_{i}$ for each iteration of the adaptive process. We can see how both, the phases and the frequencies converge to the theoretical values. In Fig. 4(b) we applied the same algorithm but using local information only. In this case, the frequencies are readjusted following $\omega_{i}^{\prime}=\omega_{i}\left(1+\left(\langle\theta\rangle_{n n}-\theta_{i}\right)\right)$, where $\langle\theta\rangle_{n n}$ is the average value of the phases of the neighbors 


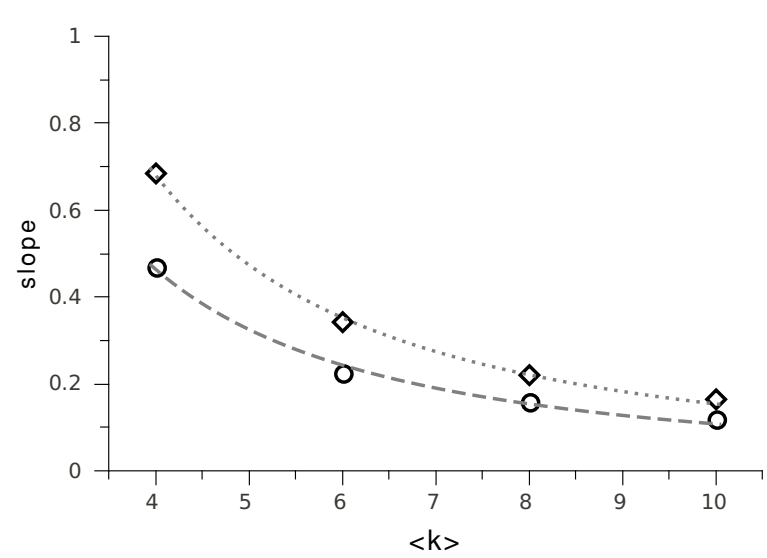

FIG. 3. Dependence of $\sigma\left(\theta_{i}\right)$ with the mean degree $\langle k\rangle$ for a distribution of the frequencies (circles) and the delays (diamonts). Lines correspond to a nolineal fitting $y=B / x^{q}$ with $B=4.22, q=1.59$ (dashed line) and $B=6.45, q=1.62$ (dotted line).

of oscillator $i$. As it can be seen from Fig. 4, both algorithms lead to perfect synchronization, however, when the frequencies are updated using local information only, a drift in the frequencies is observed (see Fig. 4(c)).

\section{LINEAL VERSUS NON-LINEAL COUPLING}

In this section we address the question of how the solution of Eq. (1) changes when a non-linear coupling between the oscillators is considered like the Kuramoto system described as

$$
\dot{\phi}_{i}(t)=\omega_{i}+\sum_{j} a_{i j} \sin \left(\phi_{j}\left(t-\tau_{i j}\right)-\phi_{i}(t)\right) .
$$

Let's illustrate how the phases and the oscillation frequency changes by analyzing the motif of three elements $(1 \leftrightarrow 2 \leftrightarrow 3)$ with $a_{i j}=1, \forall i, j$. Solving Eq. (1) for this motif, we find $\Delta \theta_{13}=0, \Delta \theta_{21}=\Delta \theta_{23}=\frac{\omega \tau}{3+4 \tau}$ and $\Omega=\frac{\omega}{1+4 / 3 \tau}$. Fig. 5 shows the phase difference $\Delta \theta_{23}$ and the oscillation frequency $\Omega$ as a function of the delay when the elements of the motif interact through either lineal or sinusoidal coupling. The solution for the nonlinear interaction remains close to the solution of the lineal problem until a critical value of the delay $\tau_{0}$ is reached. Then, for delays grater than $\tau_{0}$, a new solution of the nonlinear problem appears [21].

\section{A. Normalized interaction}

An interesting issue that is not addressed in general is the normalization in the coupling. So far we have analyzed in detail the clusterization of the phases of Eq. (1) where the influence for one neighbor adds to the influence
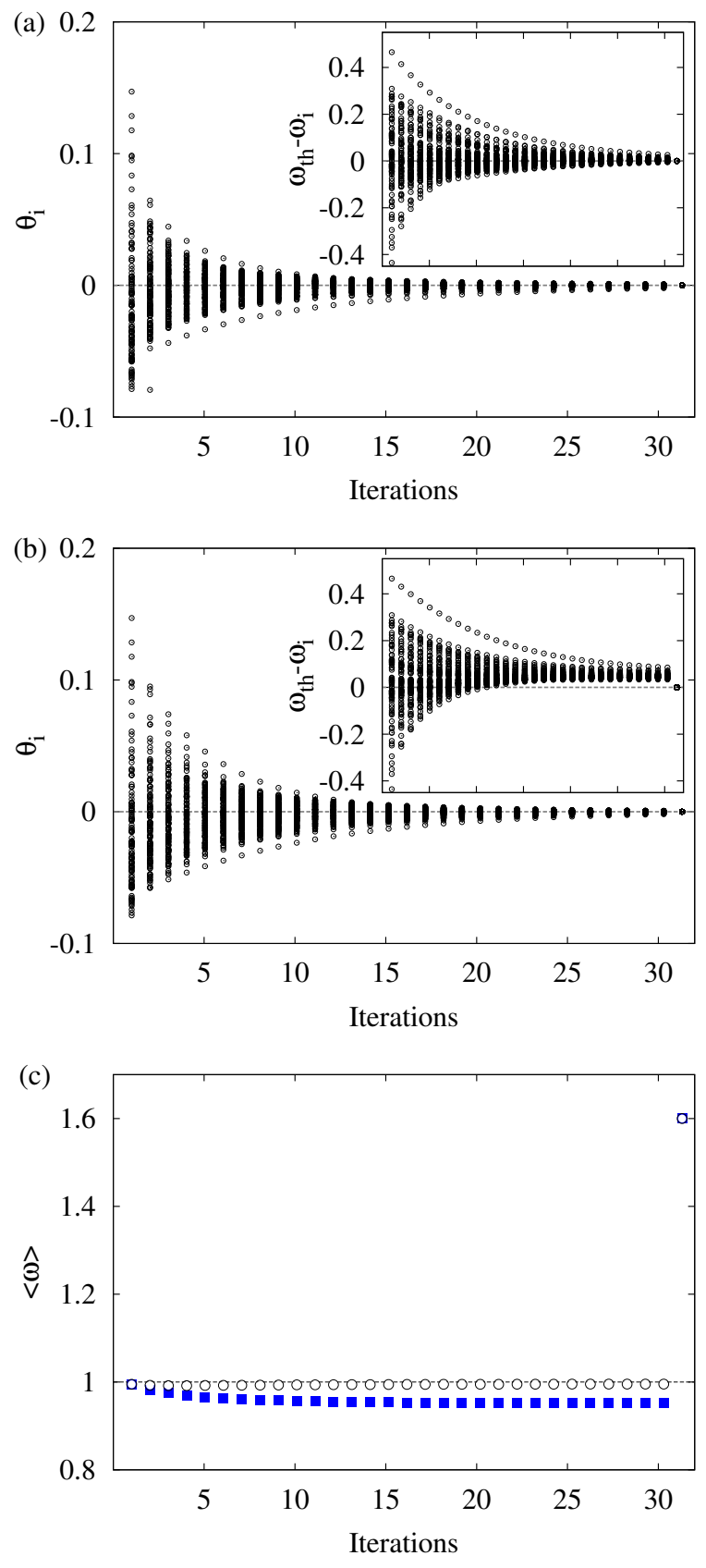

FIG. 4. Dispersion of the individual phases $\theta_{i}$ after applying the proposed algorithm using (a) global information and (b) local information. Inset: (in both panels) difference between the theoretical frequency $\omega_{t h}$ and the current frequency $\omega_{i}$ of each node. (c) Evolution of the mean frequency for the adaptive process using global (circles) and local (squares) information. The last iteration corresponds to the value of the mean frequency after apply the recipe given in Eq. (8).

of the others. Alternatively we could consider the case

$$
\dot{\phi}_{i}(t)=\omega_{i}+\frac{1}{k_{i, \text { in }}} \sum_{j} a_{i j}\left(\phi_{j}\left(t-\tau_{i j}\right)-\phi_{i}(t)\right) .
$$



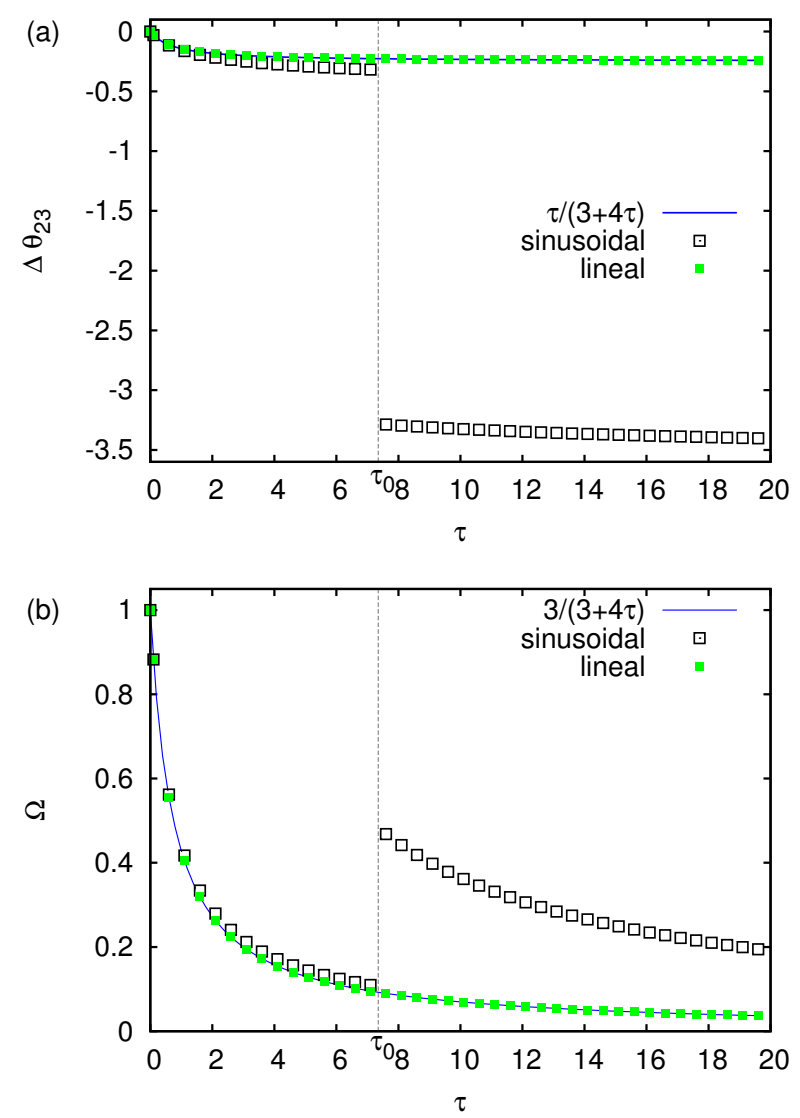

FIG. 5. (a) Phase difference $\Delta \theta_{23}$ and (b) oscillation frequency $\Omega$ as a function of the delay $\tau$ for the motif of three elements $(1 \leftrightarrow 2 \leftrightarrow 3)$ under different coupling interactions. The natural frequency of each oscillator is $\omega_{i}=1.0$.

Following the similar approach as in the previous section, a unique phase locked solution with frequency $\Omega$ exists if the phases satisfy

$$
\mathbf{k}_{\mathrm{in}} \cdot(\boldsymbol{\omega}-\Omega(\mathbf{1}+\mathbf{T}))=\mathbf{L} \boldsymbol{\theta},
$$

where $\mathbf{k}_{\text {in }}=\left(k_{1, \text { in }}, \ldots, k_{N, \text { in }}\right)$. Again, the locking frequency is given by

$$
\Omega=\frac{\left\langle\mathbf{k}_{\mathrm{in}} \boldsymbol{\omega}\right\rangle}{\langle\boldsymbol{k}\rangle+\langle\boldsymbol{T}\rangle} .
$$

Some simple cases are worth considering. For undirected networks and identical $\omega_{i}=\omega$ and $\tau_{i j}=\tau$ the locking frequency is given by

$$
\Omega=\frac{\omega}{1+\tau}
$$

and the phases are the same $\theta_{i}=\theta$, that is, independent of the underlying topology. Introducing heterogeneity in the natural frequencies and/or delays gives rise to a distribution of the phases. This is in contrast to the solution of the unnormalized solution that depends on the underlying topology even in the undirected case. Additionally, the value of the delay $\tau_{0}$ where the destabilization of the solution for the sinusoidal coupling occurs is shifted (see Fig. 6).

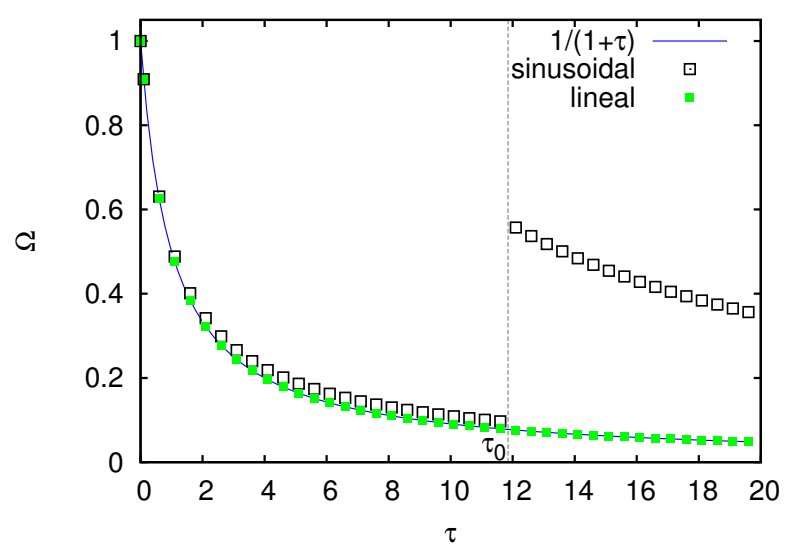

FIG. 6. Oscillation frequency $\Omega$ for the motif of three elements $(2 \leftrightarrow 1 \leftrightarrow 3)$ under different normalized interactions. The natural frequency of each oscillator is $\omega_{i}=1.0$.

We can extend our study beyond the motif structure and analyze more complex networks. We explore in Fig. 7 the effect of the averaged degree in an ER network composed of $N=10^{3}$ nodes with a mean degree $\langle k\rangle$. The solution for the nonlinear coupling is well approximated by the solution of the linearized interaction at high mean degree values, while it deviates from the lineal case at low mean degree values $(\langle k\rangle=4$ and 6$)$. The oscillation frequency $\Omega$ is also well approximated in both linear and sinusoidal coupling by Eq. (8) which solution is indicated by solid lines in Fig. 7(b).

\section{HETEROGENEOUS MEAN-FIELD APPROXIMATION}

As we have shown, for a given network we can find the locked solution. However the formal solution does not allow us to understand the role played by the different topological features in how phases are distributed. To make some understanding in this direction we perform an heterogeneous mean-field approximation for uncorrelated random networks. For the sake of clarity we will restrict ourselves to the case of undirected networks [22]. The approximation coarse-grains the dynamics to classes of nodes of the same degree $\mathbf{k}$; we define the phase density $\Phi_{\mathbf{k}}$, and the frequency density $\Omega_{k}$ of nodes of degree $k$ as

$$
\begin{array}{r}
\Phi_{\mathbf{k}}=\frac{1}{N_{\mathbf{k}}} \sum_{i \in K} \phi_{i} \\
W_{\mathbf{k}}=\frac{1}{N_{\mathbf{k}}} \sum_{i \in K} \omega_{i} .
\end{array}
$$

where $N_{\mathbf{k}}=P(\mathbf{k}) N$ is the expected number of nodes with degree $\mathbf{k}$, and $K$ denotes the set of nodes with degree $\mathbf{k}$. 

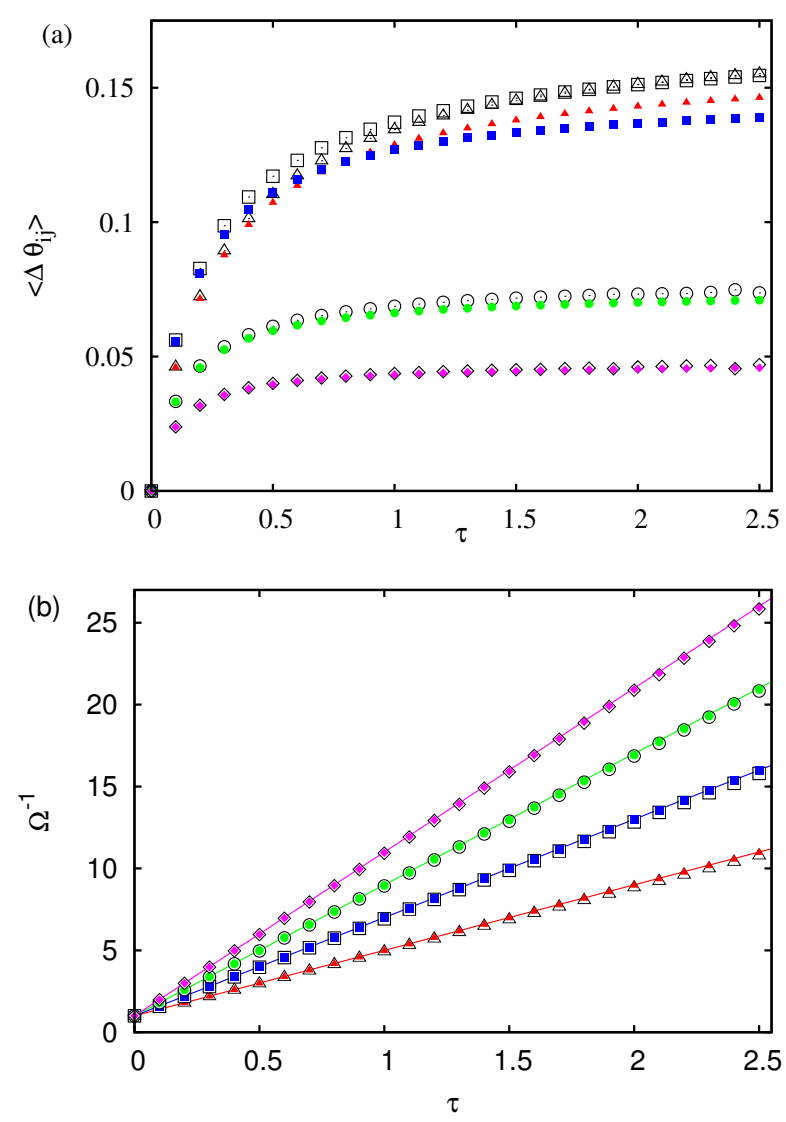

FIG. 7. (a) Averaged phase difference $\left\langle\Delta \theta_{i j}\right\rangle$ and (b) inverse of the oscillation frequency $\Omega$ in a ER network with different average degree $\langle k\rangle=4$ (triangles), 6 (squares), 8 (circles) and 10 (diamonds). Filled (open) symbols stand for lineal (sinusoidal) coupling. Other parameters: $N=10^{3}, \omega_{i}=1$ and $\tau_{i}=0.1$.

This notation allows to group the sums by the degrees of the nodes. For instance, if the degree of node $i$ is $k_{i}=k$ then

$$
\sum_{j} a_{i j} \phi_{j}=k \sum_{k^{\prime}} P\left(k^{\prime} \mid k\right) \Phi_{k^{\prime}}
$$

where $P\left(k^{\prime} \mid k\right)$ measures the probability to reach a vertex of degree $k^{\prime}$ leaving from a vertex of degree $k$. The time evolution of the phase density of the class of nodes of degree $k, \Phi_{k}(t)$ can be rewritten from Eq. (1) as

$$
\dot{\Phi}_{k}(t)=W_{k}+k \sum_{k^{\prime}} P\left(k^{\prime} \mid k\right)\left(\Phi_{k^{\prime}}(t-\tau)-\Phi_{(}(t)\right) .
$$

For uncorrelated networks $P\left(k^{\prime} \mid k\right)=\frac{k^{\prime} P\left(k^{\prime}\right)}{\langle k\rangle}$ and with the ansatz of locked solutions $\Phi_{k}=\Omega t+\Theta_{k}$, we obtain

$$
\Omega=W-\Omega T_{k}+\frac{k}{\langle k\rangle} \sum_{k^{\prime}} k_{\text {out }}^{\prime} P\left(k^{\prime}\right)\left(\Theta_{k^{\prime}}-\Theta_{k}\right) .
$$

Summing over all degrees we find

$$
\begin{gathered}
\Theta_{\mathbf{k}}=\frac{\Omega\langle T\rangle}{k}+a, \\
\Omega=\frac{\langle k \omega\rangle}{1+\langle T\rangle},
\end{gathered}
$$

being $a$ an arbitrary constant. For identical oscillators we recover for the locking frequency

$$
\Omega=\frac{\omega\langle k\rangle}{1+\langle k\rangle \tau},
$$

where $\langle k\rangle \tau=\langle\mathbf{T}\rangle$, and

$$
\Theta_{k}=\frac{\Omega\langle k\rangle \tau}{k}+a .
$$

This indicates that whether two nodes show a similar phase depends, in an uncorrelated network, on their degree difference. It also shows that low-degree nodes are ahead of high-degree nodes. Obviously, this dependence of the degree is reminiscent of our hypothesis of a meanfield coarse-grained by degree, however it is not trivial that this approximation will hold for the actual dynamics (Eq. (1)).

In Figure 8, for each node (circles), we display its degree and its phase; averaging over classes of nodes with the same degree $k$ (squares), we represent the phase $\Theta_{k}$ vs. the inverse of the degree $k^{-1}$. The analytical calculation predicts a linear dependence irrespective of the details of the degree distribution $P(k)$. The phases are obtained after integration of Eq. (1) with $\omega_{i}=1$ and $\tau_{i j}=0.1$.

\section{A. C. elegans}

The heterogeneous mean field formalism describes the relationship between dynamics and topology in uncorrelated networks. Such relationship can be illustrated in a real (correlated) network analyzing the dynamics of Eq. (1) using the connectivity of the neural system of the C. elegans. Because of its small and well-characterized nervous system and amenability to genetic manipulation, the nematode $C$. elegans offers the promise of understanding the mechanisms underlying a whole animal's behavior at the molecular and cellular levels. In fact, this goal was a primary motivation behind the development of C. elegans as an experimental organism 40 years ago. Yet it has proven surprisingly difficult to obtain a mechanistic understanding of how the C. elegans nervous system generates behavior, despite the existence of a 'wiring diagram' that contains a degree of information about neural connectivity unparalleled in any organism. This neuronal network can be represented as an adjacency matrix of 275 nonpharyngeal neurons, out of a total of 302 neurons [23]. We assume that the nervous system of the C. elegans can be modeled as a network, 

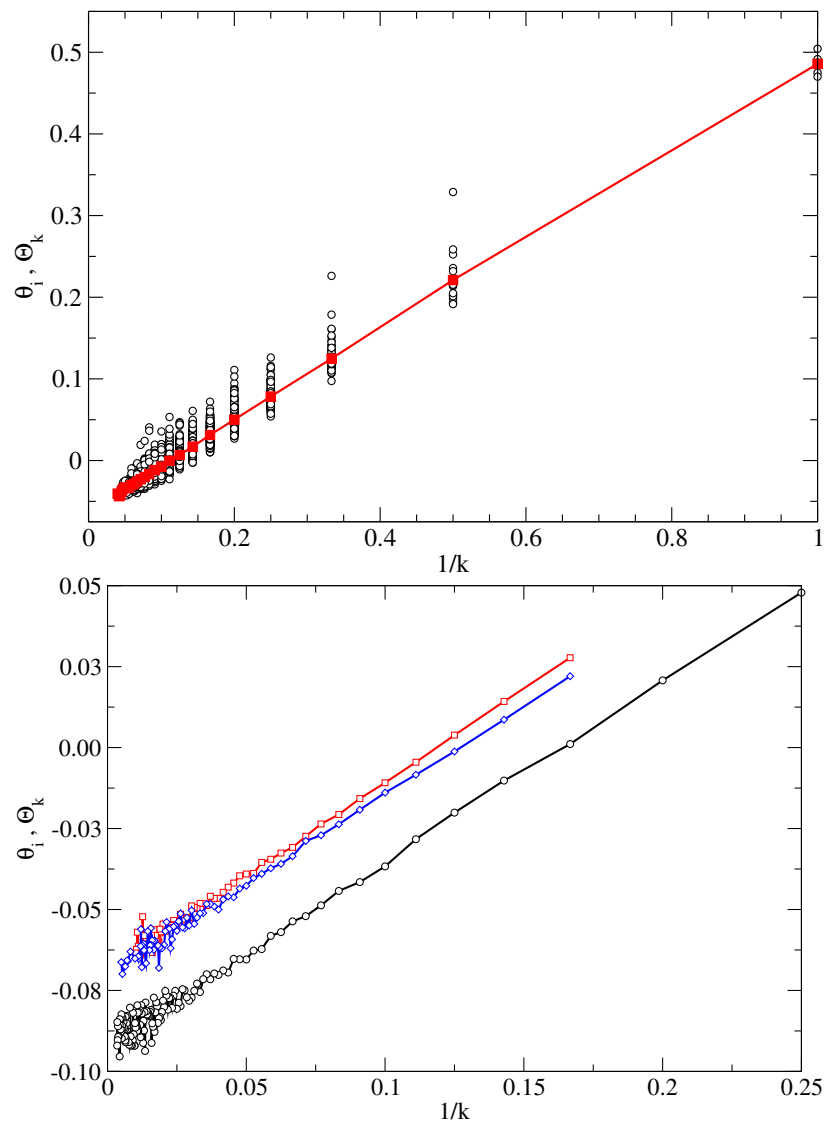

FIG. 8. Dependence of the phase of the nodes on their degree $k$ in different undirected networks: (upper panel) for an ER network with average degree $\langle k\rangle=10$; (bottom panel) for scale-free networks with different scaling exponents $(3.5,3.0$, 2.5 from top to bottom).

where nodes represent the center of the cell bodies, and the links represent synapses.

Here we propose to use the dynamical model of diffusive oscillators proposed to get a functional response (phase correlation) and after, investigate the relationship between this functional network and its topological counterpart.

In the upper panel of Fig. 9, each symbol indicates the position of a neuron and its phase obtained integrating the time delay diffusive coupled system given by Eq. (1) (open circles) and the Kuramoto-like system given by Eq. (9) (solid circles). In the bottom panel of Fig. 9 we plot the phases versus the in-degree $k_{\text {in }}$ for neurons in the C. elegans and rewired networks (averaged over 100 realizations of the rewiring algorithm) keeping the same $\left(k_{\text {in }}, k_{\text {out }}\right)$ for each neuron. Even though the network is correlated, the analytical result captures the dependence of the phase with the degree. In the rewired networks the phase is well approximated by the relation $\Theta(k)=$ $b / k+a$. The averaged phases obtained in Fig. 9 shows a good agreement with Eq. (22).

\section{B. Autonomous System Network}

Another real network that can be use to test our theory is the Internet network at the Autonomous System level. The ASP2P Internet data set considered is composed of autonomous systems (AS) [24] in the peer-topeer (P2P) category, where two ASs freely exchange traffic between themselves and their customers, but do not exchange traffic from or to their providers or other peers [25]. The network obtained results in 1217 nodes and 4058 links [26]. In Fig. 10 we show the phase of each node (circles) as a function of the inverse of its degree. The average phase $\Theta_{k}$ of classes of nodes with degree $k$ (squares) shows a good agreement with Eq. (22), denoted by the dashed line.

The relationship between the topological and functional network we have found by means of the het-
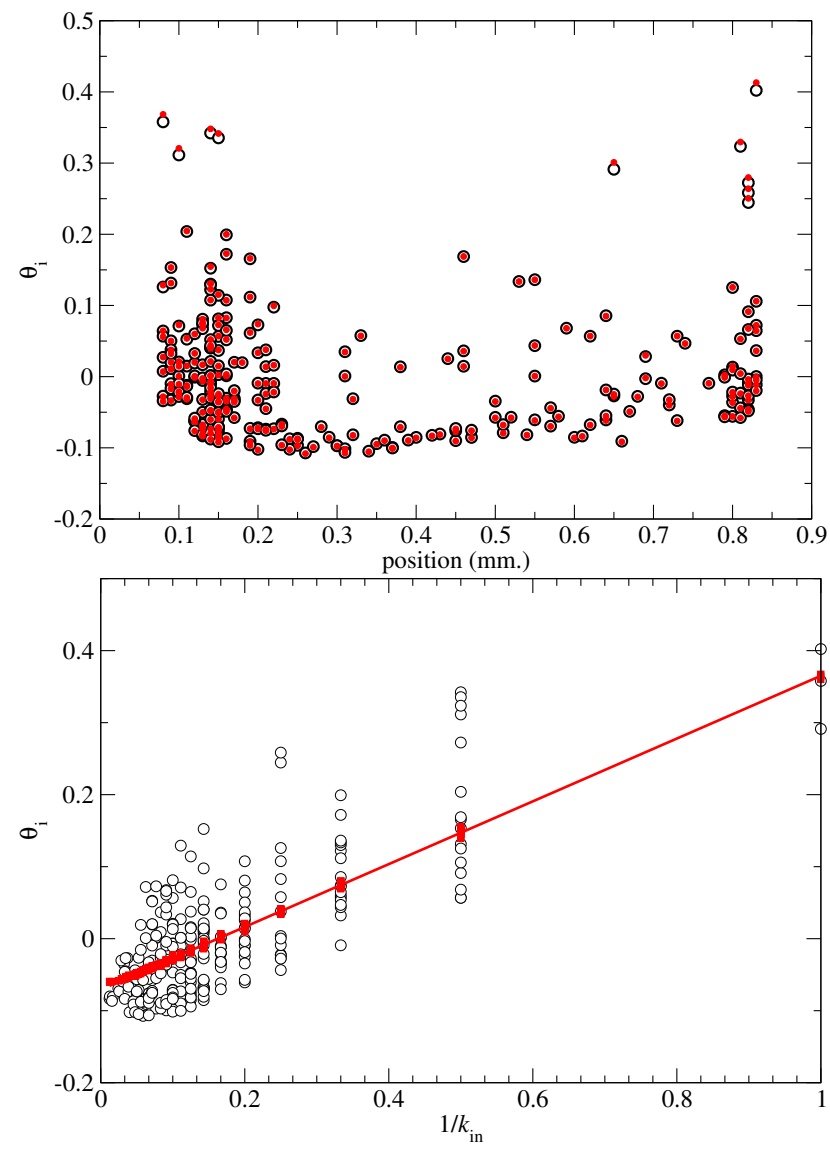

FIG. 9. (Upper panel) Nonlinear phase clusterization in the $C$. elegans. Each symbol indicates the position of a neuron and its phase (open circles) and the Kuramoto-like system where the interaction network corresponds to the directed neural network of the C. Elegans (solid symbols). (Bottom panel) Phase clusterization in the directed $C$. elegans neural network versus the in-degree $k_{\text {in }}$ for neurons in the $C$. elegans (circles) and rewired networks (squares) keeping the same $\left(k_{\text {in }}, k_{\text {out }}\right)$ for each neuron. The phases are obtained after integration of Eq. 1 with $\omega_{i}=1$ and $\tau_{i j}=0.1$. 


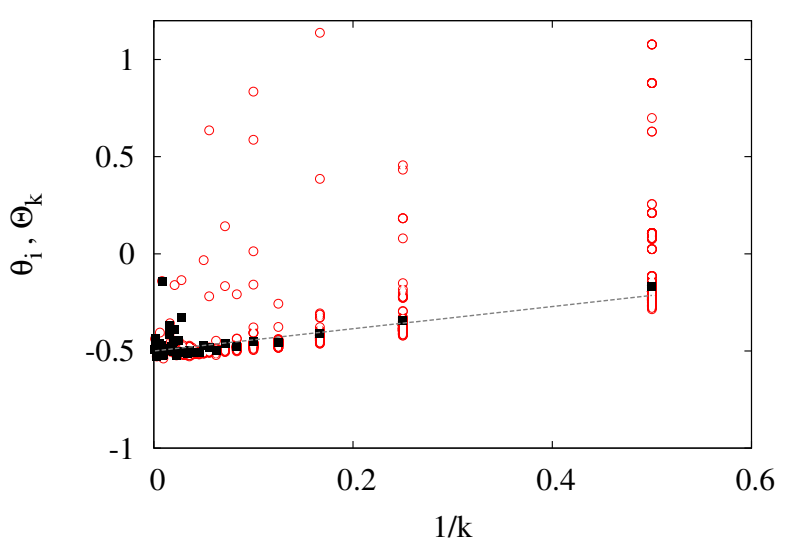

FIG. 10. Dependence of the phase $\theta_{i}$ (open ciecles) and the average phase $\Theta_{k}$ (squares) of a node on its in-degree $k$ for the Internet network at the autonomous system level. Dashed line stad for the prediction given by Eq. (22).

erogeneous mean-field approximation is in good agreement with a recent study of complex oscillator networks with non-delay interactions in the presence of noise [27]. In this case, the presence of noise allows to determine the correspondence between the dynamical correlation among the oscillators and the latent topology. Similarly to our case, a relationship of the correlated dynamics with the inverse of the degree is found for uncorrelated networks.

\section{CONCLUSIONS}

We provide the exact formal solution of a system of $N$ elements delay coupled in a network, that is, given a coupling network in Eq. 1, we solve for the locking frequency and the phases as long as the network has a single in-component. We illustrate the solution in a motif of three nodes and in an ER topology exploring in both cases the dependence on the delay and the nonlinearity within the interactions. We investigate the role played by the heterogeneities in the frequencies and the delays finding a linear relationship in the dispersion of the phases. We obtain perfect synchronization by mean of a self-adapting algorithm that dynamically adjust the frequencies. We also extend our analysis to classes of uncorrelated directed networks by means of the heterogeneous mean-field approximation. We have found the solution for the locking frequency and phases, which approximates the dynamics for these classes of networks and obtain an accurate agreement for real topologies.
[1] A. T., Winfree, J. Theor. Biol. 16, 15 (1967).

[2] A. Pikovsky, M. Rosenblum, and J. Kurths, Synchronization: A universal concept in nonlinear sciences (Cambridge University Press, Cambridge, England, 2003).

[3] S. Strogatz, Sync: The emerging science of spontaneous order (Hyperion, New York, 2003).

[4] A. Arenas, A. Díaz-Guilera, J. Kurths, Y. Moreno, and C. Zhou, Phys. Rep. 469, 93 (2008).

[5] T. Y.-C. Tsai, Y. S. Choi, W. Ma, J. R. Pomerening, C. Tang, and James E. Ferrell, Jr. Science 321, 126-129 (2008)

[6] M. Dhamala, V.K. Jirsa and M.Z. Ding, Phys. Rev. Lett. 92, 074104. (2004).

[7] D. Yu and S. Boccaletti, Phys. Rev. E 80, 036203 (2009).

[8] I. Fischer, R. Vicente, J.M. Buldu, M. Peil, C.R. Mirasso, M.C. Torrent and J. Garcia-Ojalvo, Phys. Rev. Lett. 97, 123902 (2006).

[9] R. Vicente, L.L. Gollo, C.R. Mirasso, I. Fischer and P. Gordon, Proc. Natl. Acad. Sci. USA 105, 17157 (2008).

[10] W. Kinzel, A. Englert, G. Reents, M. Zigzag and I. Kanter, Phys Rev E 79, 056207 (2009).

[11] G.L. Gerstein and A.M. Aertsen, J. Neurophysiol. 54 1513 (1985).

[12] G. Palm, A. Aertsen and G.L. Gerstein Biol. Cybern. 59 1 (1988).

[13] C. Zhou, L. Zemanova, G. Zamora, C.C. Hilgetag, J. Kurths, Phys. Rev. Lett. 97238103 (2006).

[14] D. Yu, M. Righero and L. Kocarev, Phys. Rev. Lett. 97, 188701 (2006).
[15] M. Timme, Phys. Rev. Lett. 98, 224101 (2007).

[16] I. Pajevic and D. Plenz PLoS Comp. Biol. 5, e1000271 (2008).

[17] D. Napoletani and T. D. Sauer, Phys. Rev. E 77, 026103 (2008).

[18] B. Barzel and O. Biham, Phys. Rev. E. 80, 046104 (2009).

[19] Y. Kuramoto, in Lectures notes in Physics No 30, edited by H. Araki (Springer, New York, 1975).

[20] F.R.K. Chung, CBMS Regional Conference Series in Mathematics 92, 1 (1997).

[21] D. Zanette, Phys. Rev. E 62, 3167 (2000).

[22] V.M. Eguíluz, T. Pérez, J. Borge-Holthoefer and A. Arenas. Phys. Rev. E (in press 2011)

[23] http://www.wormatlas.org/

[24] Dimitropoulos X, Krioukov D, Riley G Y and Claffy K C 2006 Revealing the autonomous system taxonomy: the machine learning approach. Passive and Active Measurements Workshop (PAM) ed S Uhlig, K Papagiannaki and O Bonaventure (Berlin: Springer)

[25] Dimitropoulos X, Krioukov D, Fomenkov M, Huffaker B, Hyun Y, Claffy K C and Riley G. Comput. ACM SIGCOMM. Commun. Rev. 3729 (2007)

[26] A. Arenas, J. Borge-Holthoefer, S. Gomez and G. Zamora-Lopez, New Journal of Physics 12, 053009 (2010)

[27] J. Ren, W.X. Wang, B. Li and Y.C. Lai, Phys. Rev. Lett. 104, $058701(2010)$ 